\title{
The Art and Science of Building a Computational Model to Understand Hemostasis
}

\author{
Karin Leiderman, $\mathrm{PhD}^{1}$ \\ Suzanne S. Sindi, $\mathrm{PhD}^{2}$ \\ Keith B. Neeves, $\mathrm{PhD}^{5}$ \\ ${ }^{1}$ Department of Applied Mathematics and Statistics, Colorado School \\ of Mines, Golden, Colorado \\ 2 Department of Applied Mathematics, University of California, \\ Merced, Merced, California \\ ${ }^{3}$ Department of Medicine, UNC Blood Research Center, University of \\ North Carolina at Chapel Hill, Chapel Hill, North Carolina \\ ${ }^{4}$ Departments of Mathematics and Biomedical Engineering, \\ University of Utah, Salt Lake City, Utah \\ ${ }^{5}$ Department of Bioengineering, Department of Pediatrics, Section of \\ Hematology, Oncology, and Bone Marrow Transplant, Hemophilia \\ and Thrombosis Center, University of Colorado, Denver, Colorado
}

Dougald M. Monroe, $\mathrm{PhD}^{3}$

Aaron L. Fogelson, $\mathrm{PhD}^{4}$

\author{
Address for correspondence Keith B. Neeves, PhD, University of \\ Colorado Anschutz Medical Campus, 12800 East 19th Avenue, Mail \\ Stop 8302, Aurora, CO 80045 \\ (e-mail: keith.neeves@cuanschutz.edu).
}

Semin Thromb Hemost 2021;47:129-138.

\begin{abstract}
Computational models of various facets of hemostasis and thrombosis have increased substantially in the last decade. These models have the potential to make predictions that can uncover new mechanisms within the complex dynamics of thrombus formation. However, these predictions are only as good as the data and assumptions they are built upon, and therefore model building requires intimate coupling with experiments. The objective of this article is to guide the reader through how a computational model is built and how it can inform and be refined by experiments.

\section{Keywords}

- blood coagulation

- platelets

- hemodynamics

- theoretical models

- computer simulation This is accomplished by answering six questions facing the model builder: (1) Why make a model? (2) What kind of model should be built? (3) How is the model built? (4) Is the model a "good" model? (5) Do we believe the model? (6) Is the model useful? These questions are answered in the context of a model of thrombus formation that has been successfully applied to understanding the interplay between blood flow, platelet deposition, and coagulation and in identifying potential modifiers of thrombin generation in hemophilia $\mathrm{A}$.
\end{abstract}

Computational models of hemostasis and thrombosis complement in vitro and in vivo models by providing new tools for understanding these phenomena. Their advantage lies in the ability to simulate and interrogate complex systems where intuition and empiricism often fail. They are able to generate new hypotheses, to simulate conditions that would be difficult or impossible to perform experimentally, to discover new mechanisms, and to explain paradoxical experimental and clinical observations in a variety of organs and pathologies. ${ }^{1}$ Pharmacokinetics/pharmacodynamics models are commonly used to predict the response to new drugs and for scaling animal models to humans. ${ }^{2}$ Network models have identified new drug targets in cancer. ${ }^{3}$ Electrophysiology models are used to simulate a wide range of
Issue Theme Personalized Approaches to Bleeding and Thrombotic Disorders; Guest Editors: Jamie O'Sullivan, PhD and Roger Preston, PhD.

DOI https://doi.org/ 10.1055/s-0041-1722861. ISSN 0094-6176. (c) 2021. The Author(s).

This is an open access article published by Thieme under the terms of the Creative Commons Attribution-NonDerivative-NonCommercial-License, permitting copying and reproduction so long as the original work is given appropriate credit. Contents may not be used for commercial purposes, or adapted, remixed, transformed or built upon. (https://creativecommons.org/ licenses/by-nc-nd/4.0/)

Thieme Medical Publishers, Inc., 333 Seventh Avenue, 18th Floor, New York, NY 10001, USA 
cardiac diseases like atrial fibrillation. ${ }^{4}$ However, computational models have yet to be widely implemented in basic or translational research in hemostasis and thrombosis. This is partly due to their descriptions being highly technical and accessible only to the experts and researchers that develop them, which hides their full potential. As a result, there is a lack of understanding of how models are built, how and when they should be applied, and importantly, their limitations. Our objective here is to demystify these approaches by walking the reader through a description of one particular model and then providing examples of how that model has led to discovery of new mechanisms in coagulation dynamics.

The first mathematical treatment of coagulation dates back to the 1950 s. $^{5}$ Many of the seminal studies that serve as the foundation for today's computational models were first developed in the 1980s and 1990s. These include models of thrombin generation, ${ }^{6,7}$ fibrinolysis, ${ }^{8}$ and platelet adhesion and aggregation. ${ }^{9-11}$ Over the last 20 years, advances in computational power and numerical methods ${ }^{12}$ have paved the way for models of cellular- and molecular-scale phenomena such as von Willebrand factor dynamics, ${ }^{13}$ platelet margination, ${ }^{14,15}$ platelet adhesion, ${ }^{16,17}$ and red blood cell microrheology. ${ }^{18}$ These phenomena clearly cannot be separated in vivo, but they are each uniquely complex and thus models are often initially developed to focus on one "subsystem" at a time. Computational advances have allowed the models to become more complex, both in terms of the biology (e.g., number of biochemical reactions and blood cells) and the computational expense necessary to simulate the systems (e.g., size of physical system and timescale). Integrating models of these different subsystems of hemostasis and thrombosis have begun, but these efforts are in their nascent stages. ${ }^{19,20}$

Belyaev et al recently published a review of computational models of thrombosis that includes an insightful analysis of current challenges, model limitations, and future needs. ${ }^{21}$ Several invited published comments on this article underscore the varying opinions on the needs, challenges, and strategies for the adoption of such models in research and clinical practice. ${ }^{22-26}$ We point the reader to this and other articles for reviews of mathematical models of hemostasis and thrombosis. ${ }^{27-30}$

In this article, we review a single mathematical model of flow-mediated coagulation and platelet deposition that we have developed, ${ }^{31,32}$ extended, ${ }^{33-35}$ and used to interpret and guide experimental studies. ${ }^{36-38}$ We provide a "behind-thescenes" view of this multidisciplinary scientific effort and describe the thought process involved with model development, validation, and application. In particular, we provide this insight in the context of the specific problem of identifying modifiers of thrombin generation in hemophilia A.

\section{Why Make a Mathematical Model?}

Mathematical models are most useful when they can answer scientific questions and, if a model does not exist to address a specific question, one could be designed with the questions in mind. In this case, we want to answer the following question: how does plasma composition alter thrombin generation during thrombus formation when factor VIII (FVIII) is deficient? This question was motivated by the significant variation in bleeding frequency and severity within clinical categories-severe, moderate, mild-of hemophilia $A,{ }^{39,40}$ which are not accurately predicted by standard laboratory assays. ${ }^{41}$ The normal ranges of coagulation factor zymogens and cofactors and endogenous anticoagulants is accepted as around $50 \%$ to $150 \%$ of the mean values of the healthy population. ${ }^{42}$ This is a remarkably broad range as compared to, for example, tightly regulated plasma ion concentrations. ${ }^{43}$ The breadth in normal factor variation suggests hemostasis is a robust system capable of fulfilling its physiologic function in the face of wide variations in its individual components.

We hypothesized that normal variations in plasma protein composition could significantly alter thrombin generation when one component, FVIII, is deficient. To systematically vary the concentrations of all the zymogens, cofactors, and endogenous anticoagulants simultaneously in an experimental system of thrombus formation under flow would be both time consuming and expensive. In vitro and in vivo flow models of thrombus formation are low throughput, even with the development of high content microfluidic devices, ${ }^{44-46}$ relative to well-plate based biochemical and cell models of thrombin generation. With a computational model we can investigate these variations efficiently, using the model as a tool to guide more pointed experimental inquiry. The model can be used to study variation in thrombin generation within a large parameter space that includes plasma composition; platelet adhesion, aggregation, and binding sites; hemodynamics, platelet margination, and mass transfer; and size and extent of injury. However, for this work to be meaningful, we must be confident that the original model captures the essential dynamics of thrombus formation.

The model we describe was first developed over 20 years ago. ${ }^{31}$ Our goal then was to build a model of thrombin generation that integrated what was known about the biochemical network of coagulation with ideas about the roles of platelets and flow. At that time, there were no experiments that looked at the system as a whole, and the model was intended to provide a tool with which to quantitatively examine the ideas in the literature about how the system functioned. Our thoughts in building the model were strongly influenced by views and data from the Mann $l a b^{47}$ on the role of surface reactions in coagulation, ideas about the essential role of platelets put forth by Monroe, Hoffman, and Roberts ${ }^{48,49}$ and by Walsh and coworkers, ${ }^{50}$ and our belief, based on the compelling studies of Turitto and coworkers, ${ }^{51,52}$ that it was essential to consider flow. The model included the biochemical network of coagulation as well as the role of platelets and blood flow in regulating this network. It was developed to answer several important questions: How does local blood flow regulate thrombin generation in the tissue factor (TF) pathway? How does binding site density on activated platelets control bursts of thrombin generation? How does platelet deposition on TF exposing subendothelium affect coagulation? This model predicted that thrombin generation under flow depended on surface 
TF concentration in a threshold manner and that small amounts of exogenous FXIa and TF worked synergistically to enhance thrombin generation. Both predictions were experimentally verified. ${ }^{37,53}$ This validation provides confidence that the model captures important qualitative dynamics of thrombus formation under flow. But perhaps more importantly, the model was also used to find the mechanisms underlying the predicted phenomena; in the case of the TF threshold, the model revealed a race between platelet-bound tenase formation and platelet coverage of the active TF surface. In the case of the TF/FXIa synergy, the model showed that the platelet-bound tenase that formed with low TF and FXIa formed quickly enough to support a thrombin burst, but did so in a unique way that specifically exploits FIX/FIXa binding sites on activated platelet surfaces. What features of this model enabled it to be a valuable tool? Its design. Designing useful models involves many decisions including the type of model, what components of the underlying system will be included, and how to incorporate them.

\section{What Kind of Model should be Built?}

Models can be grossly split into two categories: explanatory or correlative. In practice, however, most models contain elements of both. Explanatory elements are those that incorporate the mechanism of interactions between variables: For example, we know that TF forms a complex with FVIIa (see - Fig. 1 schematic). We can convert that schematic into a reaction scheme that describes the binding and unbinding of TF with FVIIa with a rate of forming the complex $\left(k_{\text {on }}\right)$ and a rate of the complex breaking apart $\left(k_{\text {off }}\right)$. The reaction scheme can

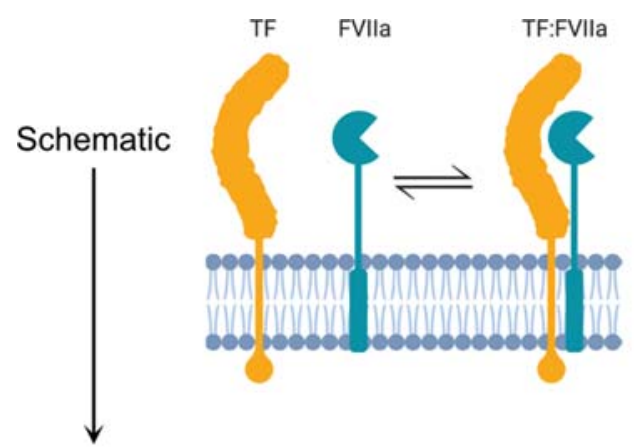

Reaction Scheme

$$
T F+F V I I a \underset{k_{o f f}}{\stackrel{k_{o n}}{\rightleftharpoons}} T F: F V I I a
$$

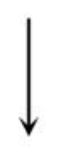

$$
\begin{aligned}
& \frac{d[T F]}{d t}=-k_{o n}[T F][F V I I a]+k_{o f f}[T F: F V I I a] \\
& \frac{d[F V I I a]}{d t}=-k_{o n}[T F][F V I I a]+k_{o f f}[T F: F V I I a] \\
& \frac{d[T F: F V I I a]}{d t}=k_{o n}[T F][F V I I a]-k_{o f f}[T F: F V I I a]
\end{aligned}
$$

Differential Equations

Fig. 1 Mathematizing biological schematics. The reaction scheme describing the binding and unbinding of activated factor VII (FVIIa) with tissue factor (TF) and the corresponding translation into mathematical equations. These equations here take the form of ordinary differential equations, meaning that they track variations in time and not in space. then be translated into mathematical equations that track how the concentrations of each of TF, FVIIa, and TF:FVIIa change in time according to the reaction scheme. These types of mathematical equations are called ordinary differential equations (ODEs). The TF:FVIIa complex converts both FIX to FIXa and FX to FXa in addition to being inhibited by TF pathway inhibitor (TFPI), and so the reaction schemes that describe all of those steps would additionally be translated into mathematical equations; in an explanatory model, equations are written for each step in the process.

By contrast, correlative elements are more like a black box, we may know inputs and outputs, but the relationships between variables are empirical or semiempirical. For example, we do not know the precise relationship between soluble agonist concentrations (adenosine diphosphate [ADP], thrombin) and platelet activation within a growing thrombus, but we do have some sense of what agonist concentration leads to activation, so we create a mathematical relationship between agonist concentration and platelet activation that emulates the desired dose-dependent activation response. Explanatory models include more detail, which may lead to complicated models (for a model of thrombus formation this can be upward of 50 equations with even more parameters), but their advantage is that they enable probing of the model for new mechanisms in a way that correlative models do not. Because we are looking for mechanistic insight into our driving question-how plasma protein composition regulates thrombin generationan explanatory model will help us to both identify the most important variables and investigate how those variables interact within the complete coagulation network. This is because an explanatory model is built using what is known about the mechanisms within the system, but the behaviors produced when these components interact emerge only from studying the full model.

Next, important features of the system we are modeling need to be considered to determine the best model for the job. The evolution of a thrombus is a dynamic process; the platelet, zymogen, enzyme, cofactor, and anticoagulant concentrations change not only in time, but also in space. Is the model dynamic or static, that is, does it change with time or not, and does it incorporate spatial components and variations? A model in which the components vary in time but do not vary in space is often called "well-mixed," since it assumes that any components are instantaneously well-mixed in space. These models are typically built using the type of ODEs shown in - Fig. 1 to evolve model components forward in time. A model that accounts for variation in space is typically built on partial differential equations to evolve the model components forward in time and track changes in space. One disadvantage of this type of model is the computational cost of tracking the spatial variations for every element of the model. For small injuries, such as those in microvascular bleeds common in hemophilia, we use a well-mixed model.

Another important question to ask is if the system is open or closed? In an open system, mass can go in and out. In a closed system, for example, modeling clotting reactions in a test tube, no mass is added or subtracted. However, as a thrombus forms under flow, blood flow transports platelets and plasma 
proteins into and out of the site of injury. To represent that with a well-mixed model, we need to assign the rate of transport for each component that is subjected to flow within the system. These rates can be derived using theories of mass transfer, ${ }^{54}$ but can be thought of more simply as being additional rate processes (written as additional terms in the mathematical equations), like in chemical reactions, that supply new reactants and carry away reaction products. In using this mass transfer assumption, we are integrating some of the physics related to blood flow without adding the computational cost of tracking spatial variations.

\section{How Is the Model Built?}

The first task in model design is deciding which variables to track with the model. Variables can include things like which coagulation proteins and inhibitors to study, the concentrations of those proteins, and which cells are included. Then it is necessary to define how the variables interact with each other. That is, for each protein in the coagulation network, we need to assign its interactions with other proteins and surfaces (endothelium, subendothelium, activated platelets). Moreover, because we are modeling the role of platelets, we also need to define how they adhere to the subendothelium, cohere with each other, and are activated by wall-bound (e.g. collagen) and soluble agonists (ADP, thrombin). This exercise requires curating the knowledge base to define these interactions as discussed in detail in the manuscripts describing our models. $^{31-33}$-Figure 2 shows a schematic of the variables' interactions considered in our model and the reaction zone where the thrombus forms.

To track how each variable changes in time due to these interactions, we need to formulate an equation for each one. Based on the decisions described above, to model thrombus formation we want a dynamic, open system that is wellmixed. Such a model can be represented by a system of ODEs. We point the reader to our book chapters that describe these types of equations in more detail. ${ }^{28,55,56}$ Briefly, ODEs consider the rate of change of a variable in time to be equal to the rates through which it participates in binding and unbinding events, chemical reactions, or mass transfer. Since many variables depend on one another, the equations for their rates of change often involve more than one of the variables. As such, the full set of ODEs is called a "coupled" system that requires the ODEs to be solved simultaneously. Fortunately, even large systems of coupled ODEs are quickly solved with a laptop computer. For example, our ODE model with 86 coupled equations simulates 20 minutes of physiological time in only seconds of computational time on a commodity computer. This makes ODE models well-suited for screening large number of conditions.

Of course, computational time is not the only criteria to consider when building a model. In order for the model to be useful it must also faithfully represent the complex biophysical and biochemical phenomena involved with thrombus formation. To accomplish both these goals requires making assumptions and approximations in our model. Basing these assumptions on experimental data is, of course, ideal but not always possible. Indeed, formulating reasonable and biologically relevant models requires experience, guess-work, luck, and conversations between researchers to understand the consequences from these assumptions. ${ }^{57} \mathrm{~A}$ few important assumptions in our model are as follows:

(1) When a platelet adheres to the subendothelium, it blocks the activity of the TF:FVIIa complex on the patch where it adheres.

(2) There are a finite number of binding sites for coagulation proteins to bind to on endothelial cells, the subendothelium, and activated platelets.

(3) The extent of injury is varied by changing the surface density of TF.

(4) Platelets are treated like a chemical species in the model, with their own mass transfer rates, that can adhere, aggregate, and activate via additional rate constants.

A full list of assumptions related to platelets, reactants, protein binding on surfaces, and transport are found in reports describing the model. ${ }^{31-33}$

The equations of the model are only one part of the modeling process. All models depend on parameters: initial conditions, biochemical rates, and physical properties of the system. Our model of coagulation under flow has 122 parameters including initial concentrations of plasma proteins, diffusion coefficients, reaction and binding rate constants, the number of binding sites on various surfaces, and rates associated with platelet adhesion, cohesion, and activation. In comparison to other modeled systems, for example, intracellular signal transduction networks, coagulation is fairly well-characterized; the plasma concentrations of each protein (and often their amount in platelet granules) are known. Moreover, the network of protein-protein interactions is well defined, and the kinetic rate constants for most protein-protein interactions have been measured in the fluid phase and on the surface of phospholipids, as appropriate. However, not all rate constants have been measured and, when model assumptions and approximations are made, new parameters are sometimes created that are not measurable. In these cases, parameters must be estimated. Finally, it is important to understand how the model behaves in response to changes in individual parameters or groups of parameters. We discuss parameter estimation and parameter sensitivity in the "Do we believe the model?" section.

\section{Is the Model a "Good" Model?}

With a model of a complex biological system in hand, we need to do a series of diagnostic tests to make sure it is well behaved, similar to what one might do with an electronic circuit. A good first test is to see if the model is internally consistent, which in the case of a protein-protein network means there is conservation of mass. For each component, the amount coming into the system minus the amount leaving the system should equal the amount generated plus the amount that accumulates (in - out = generation + accumulation). Model components go in and out of the system by blood flow-mediated transport; model components can be generated or consumed by chemical reactions; and model components can accumulate by binding to surfaces like those of activated platelets. 


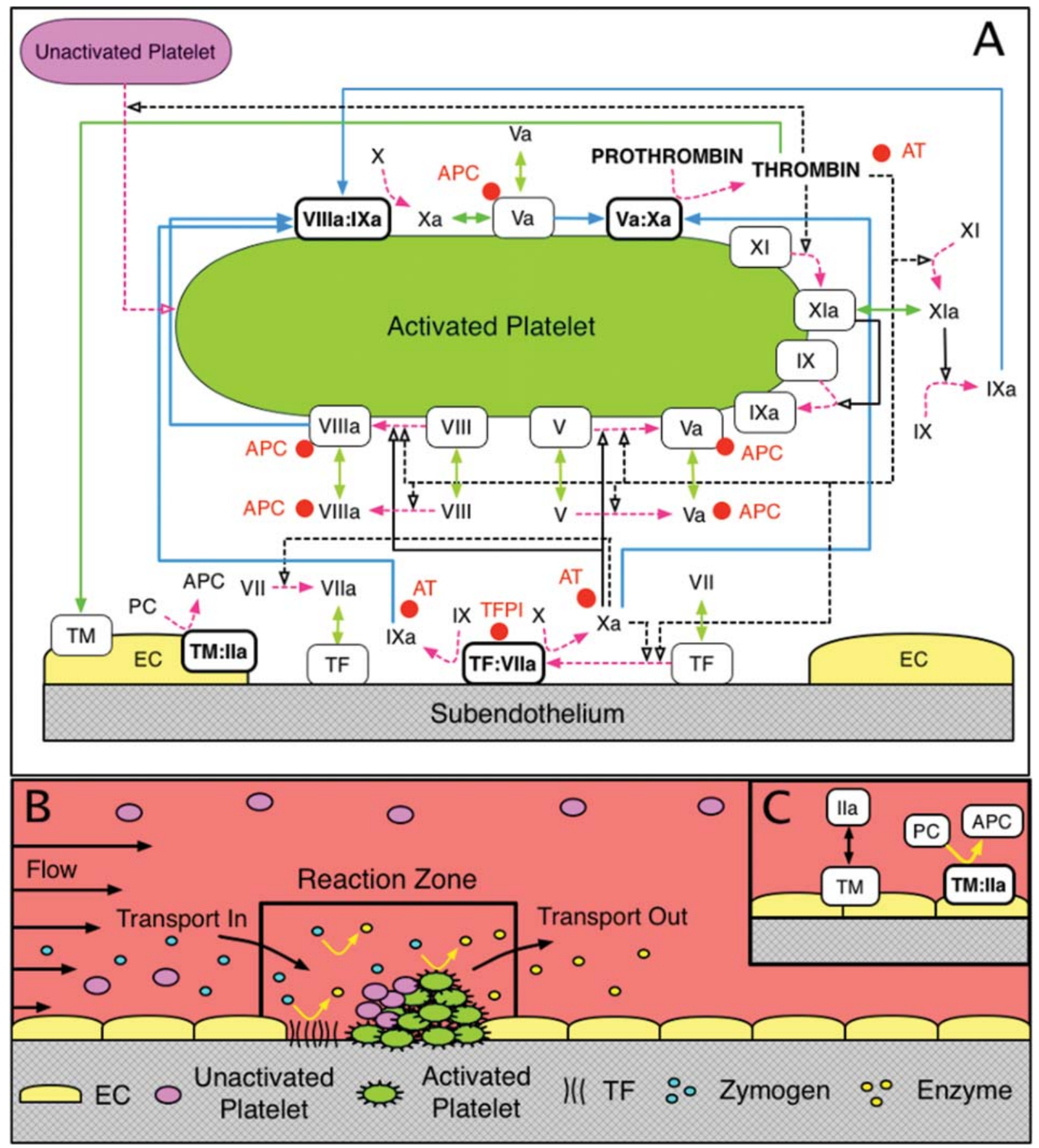

Fig. 2 (Color online) Schematic of coagulation reactions included in our model. Schematic (A) of the reaction zone where platelet deposition and coagulation reactions are tracked, and (B) of the endothelial zone into which thrombin can diffuse from the reaction zone, and in which thrombin binds to thrombomodulin and produces activated protein C (APC) which can diffuse into the reaction zone. (C) Dashed magenta arrows show cellular or chemical activation processes. Blue arrows show chemical transport in the fluid or on a surface. Green segments with two arrowheads depict binding and unbinding from a surface. Rectangular boxes denote surface-bound species. Solid black lines with open arrows show enzyme action in a forward direction, while dashed black lines with open arrows show feedback action of enzymes. Red disks show chemical inhibitors. APC, activated protein C; AT, antithrombin; EC, endothelial cell; PC, protein C; TF, tissue factor; TM, thrombomodulin. Image Courtesy: Link et al. ${ }^{64}$

The next step is to see if the model is externally consistent; that is, how close are the model results to existing data? Our model tracks the kinetics of thrombus formation on a small patch of exposed endothelium, so it makes sense to compare its output to the kinetics of experimental models of small, intravascular injuries. Using in vitro flow assays for such a comparison is the most direct because flow rates and surface
TF concentrations are user-defined parameters. Using a platelet-bound thrombin sensor, Welsh et al report a thrombin burst between 4 and 5 minutes in whole blood flow assays on collagen-TF surfaces at $100 \mathrm{~s}^{-1} .{ }^{58}$ We note this is comparable to the timescale for a thrombin burst in our model under similar conditions. In addition, in both the flow assay and the model, the thrombin burst is preceded by rapid 
accumulation of platelets within the first 2 to 3 minutes. The kinetics of platelet and fibrin accumulation and thrombin generation also occur on similar timescales in the laser injury and pipette injury models in mice. ${ }^{59,60}$ Our model predicts a steady, nearly linear increase in activated platelets over time, in agreement with the increase in P-selectin positive platelets in these animal models. Importantly, our model does not depict spatial variability, so it cannot depict the core-shell structure observed in these experimental models. However, we have developed other computational models that include spatial variations and recapitulate the observation where the core-shell structure emerges based on transport limitation. ${ }^{34,35,61}$

Other tests of external consistency include varying model inputs like platelet count and coagulation factor levels within the model and comparing model outputs to observations. Severe thrombocytopenia in the model results in a drastic drop in platelet accumulation and thrombin generation. Severe deficiencies in FVIII or FIX in the model delay the onset of thrombin generation, as expected, but a decreased maximum thrombin concentration is only observed in the model when platelet deposition blocks TF:FVIIa activity.

\section{Do We Believe the Model?}

How much confidence do we have in the model output? Mathematically, this question is phrased in terms of model uncertainty and is studied with sensitivity analysis. Because any complex model is necessarily the consequence of observations in different settings and parameter estimates from different labs, we want to understand how uncertainty in model inputs (parameters, biophysical characteristics, and initial conditions) impacts the model output. In particular, if small changes in any model input lead to large changes in model output this suggests our model is particularly sensitive to these values. Of course, uncertainty in model inputs is inevitable and there are many sources of uncertainty. There may be uncertainty in the kinetic rate constants due to the experimental conditions they were measured under. There may be uncertainty in plasma levels of clotting factors based on the broad levels of variation in those plasma factors among individuals. And, there may also be uncertainty introduced by the model formulation itself. For example, if the biochemical reaction scheme is missing true interactions our model will not be depicting the right biochemical dynamics. In addition, if we are using a system of ODEs, we will be neglecting potentially important spatial variation through diffusion or flow. Here, we assume high confidence in the model and want to quantify the uncertainty inherent in the model that comes from a lack of knowledge about kinetic parameters and initial conditions.

In this context, we are interested in studying how the uncertainty propagates through the model, from input to output. As we will describe in greater detail this enables us to not only precisely characterize the robustness of the model output (in expected ways) but to also attribute variation in model outputs (i.e., thrombin concentration) to specific model inputs (i.e., biochemical parameters, initial conditions). This type of analysis is particularly informative when the uncertainty of model inputs has been characterized. For example, as we described above, the normal ranges of coagulation factors vary over a significant range, and in our studies we consider each to vary between $50 \%$ and $150 \%$ of their mean value. As such, a reasonable investigation of our coagulation model would be to vary a clotting factor's plasma level (initial conditions in our model) and quantify the extent to which the model output is changed. Our model gives output in the form of concentrations for every chemical species in time, but we are primarily interested in the impact on thrombin generation. We may also want to study specific output metrics related to thrombin generation such as the time until some desired amount of thrombin is generated, the maximum thrombin concentration generated over some amount of time, or the maximum rate of thrombin generation.

Broadly speaking, there are two approaches to studying the sensitivity of model output to model inputs. In local sensitivity analysis, we study the sensitivity of the model output to each input on its own by varying each input over some specified range. In global sensitivity analysis, we study the sensitivity of the model output as all parameters of interest are varied simultaneously. Both forms of sensitivity analysis have been productively used on models of complex biological processes, and on coagulation in particular. However, because global sensitivity analysis tells us the relationship between multiple parameters it is able to identify combinations of proteins to which the model output is especially sensitive. ${ }^{62}$

Uncertainty quantification and sensitivity analysis are often used to test model robustness. Here we mean that since hemostasis is a robust system in healthy persons, our model should emulate this. To test for robustness and overall sensitivity of our model, we performed both a local and global sensitivity analysis on the model output metrics described above because they relate to clinical assay outputs, that is, thrombin lag time, maximum relative rate of generation, and concentration after a specified time. ${ }^{38}$ Varying plasma levels of proteins within their normal range of $50 \%$ to $150 \%$ led to strong thrombin generation within 3 to 5 minutes, which gave us confidence in model robustness (-Fig. 3). Variations in kinetic rate constants between $50 \%$ and $150 \%$ of literature-derived values led to more serious variations in output, and in some cases led to little or no thrombin generation. Not all rate constants have been measured and those that have been measured have been done under a variety of conditions using a variety of techniques that likely affect their measured values, so there is some uncertainty in their "true" values in vivo.

\section{Is the Model Useful?}

In addition to testing model robustness, sensitivity analysis of model parameters within known ranges can also be used to make predictions. Let us return to our hypothesis that normal variations in plasma protein levels can significantly modify thrombin generation in hemophilia A. We can perform a similar global sensitivity analysis in which the plasma protein levels of clotting factors were varied simultaneously 

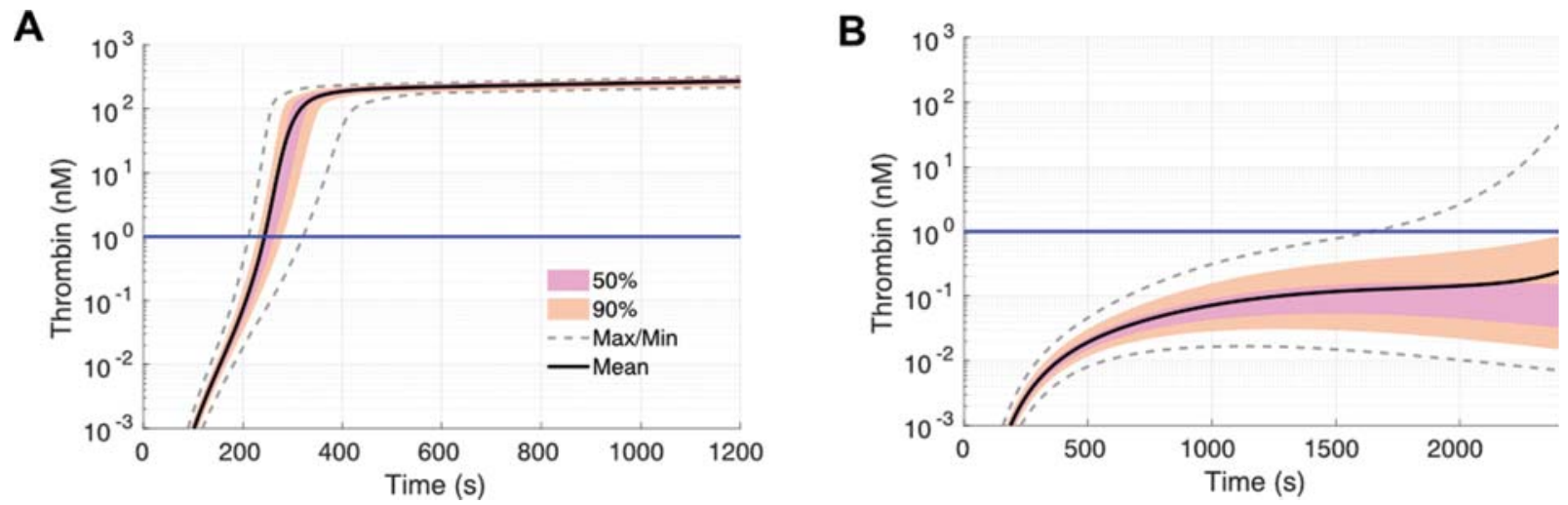

Fig. 3. (Color online) Thrombin concentration dynamics under flow generated by varying plasma zymogen and anticoagulant levels under normal (A) and severe factor VIII (FVIII) deficiency (B) conditions. Data represents 110,000 simulations where levels were uniformly varied from $50 \%$ to $150 \%$ of the population mean values. The mean (solid black line) and boundaries that encompass $50 \%$ of the data (pink), and $90 \%$ of the data (orange), and the maximum/minimum of all solutions (gray-dashed). The surface tissue factor concentration in (A) is 15 and in (B) is $5 \mathrm{fmol} / \mathrm{cm}^{2}$. Image Courtesy: Link et al. ${ }^{38,64}$

but the FVIII level was fixed to be low ( $1 \%$ of normal). We used $1 \mathrm{nM}$ as a critical thrombin concentration because it can activate platelets through protease-activated receptor $1 .^{63}$ The result of this was the prediction that prothrombin and FV levels have the strongest effect on thrombin generation when FVIII is low (1\%). ${ }^{64}$ As one might intuit, high prothrombin levels were associated with increased thrombin generation. Surprisingly, low FV levels in the range of $50 \%$ to $70 \%$ were necessary to push thrombin concentrations above $1 \mathrm{nM}$ in the model, while prothrombin levels near the high end of the normal range enhanced this effect. We verified the model's unexpected prediction with an in vitro flow assay that is an experimental analog to the model. In those assays, whole blood samples from individuals with FVIII deficiencies were perfused over a collagen-TF surface. Treatment with a partial function-blocking antibody against FV resulted in significant fibrin deposition, which was further enhanced by adding exogenous prothrombin.

With the model prediction experimentally verified, we now want to use our model as a tool that helps discover possible mechanisms. Studying the model in greater detail revealed a mechanism that might explain the counterintuitive result that low normal levels of FV could enhance thrombin generation in hemophilia: substrate competition for FXa. We have since hypothesized two other possible mechanisms (-Table 1): inhibition of FVIIIa by activated protein C (APC) and TFPI $\alpha$ associated with FV. The mechanism revealed by the model is a consequence of the fact that the initial FXa generated by TF: FVIIa has two substrates, FV and FVIII. When FV levels are reduced from normal to low-normal, more FXa is available to convert more FVIII to FVIIIa. This in turn results in more FVIIIa: FIXa, which yields more FXa and subsequently FVa:FXa, ultimately producing more thrombin. The second potential mechanism is a consequence of FV's role as a cofactor, along with protein S, for APC in FVIIIa degradation in the tenase (FVIIIa:FIXa) complex. ${ }^{65}$ In this mechanism, reduced FV levels would result in less FVIIIa degradation and consequently more thrombin generation. Finally, the third potential mechanism stems from reports that TFPI $\alpha$ may be associated with circulat- ing FV. ${ }^{66,67}$ In this mechanism, reduced FV levels would result in reduced TFPI $\alpha$ and thus reduced inhibition of TF:FVIIa and FVa:FXa. The FXa substrate competition mechanism is what we predicted from the model in its current state. Although our model currently includes APC inhibition of FVIIIa and FVa, and TFPI inhibition of FXa and thus TF:VIIa, it does not consider APC inhibition of FVa and FVIIla while they are in the tenase or prothrombinase complex nor TFPI inhibition of prothrombinase formation. As such, the model has revealed several mechanisms to study in future research.

\section{The Goals and Value of Computational Models}

The primary goal of the type of models described in this article is to make predictions, not to merely agree with existing experimental observations. If the model predictions are borne out in experiments, then the model quantitatively describes the system for a certain set of conditions. All models eventually fail to be externally consistent for some set of conditions, and at that point they must be revised. However, discrepancies between model results and experimental observations should not be viewed as a failure of a model, but rather as a seed for new discovery. The conversation between the models and experiments results in new questions that, in many cases, would not arise otherwise. Moreover, a useful model need not be consistent with every feature of the experimental data to be powerful. Our model of coagulation under flow does not include every known detail of coagulation biochemistry and platelet biology, yet it has made several interesting, and in some cases counterintuitive predictions that were verified by experiments. Even more exciting, in our opinion, is that the model predictions have motivated new and unexplored lines of inquiry. Indeed, part of the art of model building lies in the tension between providing adequate detail to describe the underlying physics, chemistry, and biology without descending into a level of complexity that is computationally intractable or stretches beyond the limits of what can be approximated quantitatively using existing knowledge. 
Table 1 Summary of potential mechanisms that explain how low normal FV enhances thrombin generation in hemophilia A

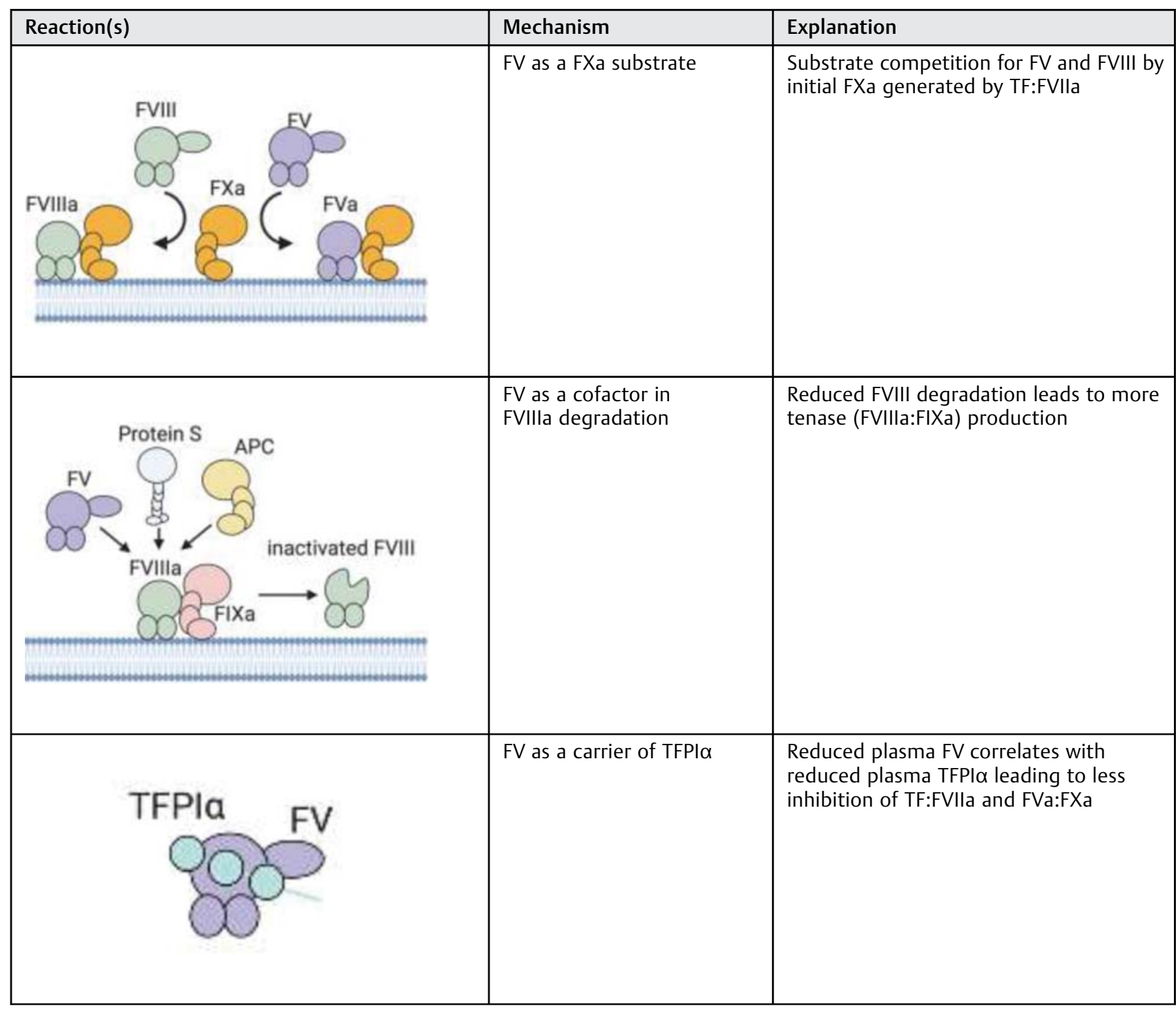

Abbreviations: APC, activated protein C; FV, factor V; TFPI, tissue factor pathway inhibitor.

\section{Conclusion}

We have highlighted the benefits of taking a computational approach to studying hemostasis in several ways. First, the novel prediction that low normal FV levels enhanced thrombin generation in hemophilia $\mathrm{A}$ was made with a computational model that was verified in experimental models. Second, a new mechanism was proposed, that remains to be verified experimentally. Third, new questions arose around the pro- and anticoagulant roles of FV in the context of hemophilia. Fourth, our study motivated the consideration of additional interactions that will be built into future versions of the model, which will only refine it and enable it to further contribute to understanding the relative importance of these three mechanisms under different conditions. This demonstrates the power of computational modeling and sensitivity analysis to study hemostasis.

We believe that the key to our successes has, in part, been the faithful integration of knowledge about biochemical and biophysical processes on many scales and in a computationally tractable way; this careful modeling enabled us to use the model itself as a tool to make predictions and hypothesize new mechanisms that motivate future experimental studies. Additionally, we stress the importance of working in a multidisciplinary team where ideas, expertise, data, and intuition are openly shared. We believe that this type of collaborative work is likely to continue yielding success in predictive and mechanistic studies of the hemostatic system.

Note

-Figure 1 and - Table 1 schematic were created with BioRender.com.

\section{Funding}

This work was supported in part by the National Institutes of Health (R01HL120728, R01HL151984), the National Science Foundation (CBET-1351672, DMS-1848221), and the Army Research Office (ARO-12369656). 


\section{Conflict of Interest}

K.B.N. reports grants from National Institutes of Health, during the conduct of the study. D.M.M. reports grants from Army Research Office, during the conduct of the study.

\section{References}

1 Winslow RL, Trayanova N, Geman D, Miller MI. Computational medicine: translating models to clinical care. Sci Transl Med 2012;4(158):158rv11

2 Wang S, Guo P, Wang X, Zhou Q, Gallo JM. Preclinical pharmacokinetic/pharmacodynamic models of gefitinib and the design of equivalent dosing regimens in EGFR wild-type and mutant tumor models. Mol Cancer Ther 2008;7(02):407-417

3 Miller ML, Molinelli EJ, Nair JS, et al. Drug synergy screen and network modeling in dedifferentiated liposarcoma identifies CDK4 and IGF1R as synergistic drug targets. Sci Signal 2013;6 (294):ra85-ra85

4 Trayanova NA. Mathematical approaches to understanding and imaging atrial fibrillation: significance for mechanisms and management. Circ Res 2014;114(09):1516-1531

5 Jurgens J. The exponentiator; a nomogram for uniform quick percentile determination of the blood coagulation factors [in German]. Arztl Wochensch 1954;9(18):420-422

6 Nesheim ME, Tracy RP, Mann KG. "Clotspeed," a mathematical simulation of the functional properties of prothrombinase. J Biol Chem 1984;259(03):1447-1453

7 Hockin MF, Jones KC, Everse SJ, Mann KG. A model for the stoichiometric regulation of blood coagulation. J Biol Chem 2002;277(21):18322-18333

8 Diamond SL, Anand S. Inner clot diffusion and permeation during fibrinolysis. Biophys J 1993;65(06):2622-2643

9 Sorensen EN, Burgreen GW, Wagner WR, Antaki JF. Computational simulation of platelet deposition and activation: I. Model development and properties. Ann Biomed Eng 1999;27(04): 436-448

10 Fogelson AL. A mathematical model and numerical method for studying platelet adhesion and aggregation during blood clotting. J Comput Phys 1984;56:111-134

11 Fogelson AL. Continuum models of platelet aggregation: formulation and mechanical properties. SIAM J Appl Math 1992; 52:1089-1110

12 Freund JB. Numerical simulation of flowing blood cells. Annu Rev Fluid Mech 2013;46:130812142306005

13 Schneider SW, Nuschele S, Wixforth A, et al. Shear-induced unfolding triggers adhesion of von Willebrand factor fibers. Proc Natl Acad Sci U S A 2007;104(19):7899-7903

14 Zhao H, Shaqfeh ESG, Narsimhan V. Shear-induced particle migration and margination in a cellular suspension. Phys Fluids 2012;24:011902

15 Crowl L, Fogelson AL. Analysis of mechanisms for platelet nearwall excess under arterial blood flow conditions. J Fluid Mech 2011;676:348-375

16 Mody NA, King MR. Platelet adhesive dynamics. Part I: characterization of platelet hydrodynamic collisions and wall effects. Biophys J 2008;95(05):2539-2555

17 Mody NA, King MR. Platelet adhesive dynamics. Part II: high shear-induced transient aggregation via GPIbalpha-vWF-GPIbalpha bridging. Biophys J 2008;95(05):2556-2574

18 Fedosov DA, Caswell B, Karniadakis GE. A multiscale red blood cell model with accurate mechanics, rheology, and dynamics. Biophys J 2010;98(10):2215-2225

19 Wang W, King MR. Multiscale modeling of platelet adhesion and thrombus growth. Ann Biomed Eng 2012;40(11):2345-2354

20 Flamm MH, Colace TV, Chatterjee MS, et al. Multiscale prediction of patient-specific platelet function under flow. Blood 2012;120 (01):190-198
21 Belyaev AV, Dunster JL, Gibbins JM, Panteleev MA, Volpert V. Modeling thrombosis in silico: frontiers, challenges, unresolved problems and milestones. Phys Life Rev 2018;26-27:57-95

22 Hemker HC, Bloemen S, Hemker PW. Exploring the limits of modelling thrombus formation: comment on "Modeling thrombosis in silico: frontiers, challenges, unresolved problems and milestones" by A.V. Belyaev et al. Phys Life Rev 2018;2627:100-105

23 Fedosov DA. Hemostasis is a highly multiscale process: comment on "Modeling thrombosis in silico: frontiers, challenges, unresolved problems and milestones" by A. V. Belyaev et al. Phys Life Rev 2018;26-27:108-109

24 Tomaiuolo M, Brass LF. Joining forces to understand hemostasis and thrombosis: a call to communicate: comment on "Modeling thrombosis in silico: frontiers, challenges, unresolved problems and milestones" by A.V. Belyaev et al. Phys Life Rev 2018;2627:110-112

25 Andrews RK, Gardiner EE. Monitoring the pulse of thrombus formation: comment on "Modeling thrombosis in silico: frontiers, challenges, unresolved problems and milestones" by A.V. Belyaev et al. Phys Life Rev 2018;26-27:113-115

26 Yazdani A, Karniadakis G. Moving toward realistic models: comment on "Modeling thrombosis in silico: frontiers, challenges, unresolved problems and milestones" by A.V. Belyaev et al. Phys Life Rev 2018;26-27:96-99

27 Leiderman K, Fogelson A. An overview of mathematical modeling of thrombus formation under flow. Thromb Res 2014;133 (Suppl 1):S12-S14

28 Neeves KB, Leiderman K. Mathematical Models of Hemostasis Trauma Induced Coagulopathy. In: Gonzalez E, Moore HB, Moore EE, eds. Trauma Induced Coagulopathy. Cham, Switzerland: Springer; 2015

29 Fogelson AL, Neeves KB. Fluid mechanics of blood clot formation. Annu Rev Fluid Mech 2015;47:377-403

30 Diamond SL. Systems analysis of thrombus formation. Circ Res 2016;118(09):1348-1362

31 Kuharsky AL, Fogelson AL. Surface-mediated control of blood coagulation: the role of binding site densities and platelet deposition. Biophys J 2001;80(03):1050-1074

32 Fogelson AL, Tania N. Coagulation under flow: the influence of flowmediated transport on the initiation and inhibition of coagulation. Pathophysiol Haemost Thromb 2005;34(2-3):91-108

33 Fogelson AL, Hussain YH, Leiderman K. Blood clot formation under flow: the importance of factor XI depends strongly on platelet count. Biophys J 2012;102(01):10-18

34 Leiderman K, Fogelson AL. Grow with the flow: a spatial-temporal model of platelet deposition and blood coagulation under flow. Math Med Biol 2011;28(01):47-84

35 Leiderman K, Fogelson AL. The influence of hindered transport on the development of platelet thrombi under flow. Bull Math Biol 2013;75(08):1255-1283

36 Onasoga-Jarvis AA, Leiderman K, Fogelson AL, et al. The effect of factor VIII deficiencies and replacement and bypass therapies on thrombus formation under venous flow conditions in microfluidic and computational models. PLoS One 2013;8(11):e78732

37 Leiderman K, Chang WC, Ovanesov M, Fogelson AL. Synergy between tissue factor and exogenous factor XIa in initiating coagulation. Arterioscler Thromb Vasc Biol 2016;36(12): 2334-2345

38 Link KG, Stobb MT, Di Paola J, et al. A local and global sensitivity analysis of a mathematical model of coagulation and platelet deposition under flow. PLoS One 2018;13(07):e0200917

39 Aledort LM, Haschmeyer RH, Pettersson HThe Orthopaedic Outcome Study Group. A longitudinal study of orthopaedic outcomes for severe factor-VIII-deficient haemophiliacs. J Intern Med 1994; 236(04):391-399

40 Blanchette VS, Key NS, Ljung LR, Manco-Johnson MJ, van den Berg HM, Srivastava ASubcommittee on Factor VIII, Factor IX and Rare 
Coagulation Disorders of the Scientific and Standardization Committee of the International Society on Thrombosis and Hemostasis. Definitions in hemophilia: communication from the SSC of the ISTH. J Thromb Haemost 2014;12(11):1935-1939

41 Watson HG, Greaves M. Can we predict bleeding? Semin Thromb Hemost 2008;34(01):97-103

42 Butenas S, van't Veer C, Mann KG. "Normal” thrombin generation. Blood 1999;94(07):2169-2178

43 Andersen OS. Cellular electrolyte metabolism. Encyclopedia of Metalloproteins New York: Springer; 2013:580-587

44 Maloney SF, Brass LF, Diamond SL. P2Y12 or P2Y1 inhibitors reduce platelet deposition in a microfluidic model of thrombosis while apyrase lacks efficacy under flow conditions. Integr Biol 2010;2(04):183-192

45 Hansen RR, Wufsus AR, Barton ST, Onasoga AA, Johnson-Paben RM, Neeves KB. High content evaluation of shear dependent platelet function in a microfluidic flow assay. Ann Biomed Eng 2013;41(02):250-262

46 de Witt SM, Swieringa F, Cavill R, et al. Identification of platelet function defects by multi-parameter assessment of thrombus formation. Nat Commun 2014;5:4257

47 Mann KG, Nesheim ME, Church WR, Haley P, Krishnaswamy S. Surface-dependent reactions of the vitamin K-dependent enzyme complexes. Blood 1990;76(01):1-16

48 Monroe DM, Roberts HR, Hoffman M. Platelet procoagulant complex assembly in a tissue factor-initiated system. Br J Haematol 1994;88(02):364-371

49 Hoffman M, Monroe DM, Roberts HR. Cellular interactions in hemostasis. Haemostasis 1996;26(Suppl 1):12-16

50 Ahmad SS, Rawala-Sheikh R, Walsh PN. Comparative interactions of factor IX and factor IXa with human platelets. J Biol Chem 1989; 264(06):3244-3251

51 Weiss HJ, Baumgartner HR, Turitto VT. Regulation of plateletfibrin thrombi on subendothelium. Ann N Y Acad Sci 1987; 516:380-397

52 Nemerson Y, Turitto VT. The effect of flow on hemostasis and thrombosis. Thromb Haemost 1991;66(03):272-276

53 Okorie UM, Denney WS, Chatterjee MS, Neeves KB, Diamond SL. Determination of surface tissue factor thresholds that trigger coagulation at venous and arterial shear rates: amplification of $100 \mathrm{fM}$ circulating tissue factor requires flow. Blood 2008;111(07):3507-3513

54 Rana K, Neeves KB. Blood flow and mass transfer regulation of coagulation. Blood Rev 2016;30(05):357-368
55 Leiderman K, Bannish BE, Kelley MA, Palmisano AM. Mathematical models of thrombus formation and fibrinolysis. In: Topaz O, ed. Cardiovascular Thrombus: From Pathology and Clinical Presentations to Imaging, Pharmacotherapy and Interventions. San Diego, United States Academic Press; 2018:67-86

56 Banwarth-Kuhn M, Sindi S. How and why to build a mathematical model: a case study using prion aggregation. J Biol Chem 2020; 295(15):5022-5035

57 Reed MC. Why is mathematical biology so hard? Not Am Math Soc 2004;51:338-342

58 Welsh JD, Colace TV, Muthard RW, Stalker TJ, Brass LF, Diamond SL. Platelet-targeting sensor reveals thrombin gradients within blood clots forming in microfluidic assays and in mouse. J Thromb Haemost 2012;10(11):2344-2353

59 Stalker TJ, Traxler EA, Wu J, et al. Hierarchical organization in the hemostatic response and its relationship to the platelet-signaling network. Blood 2013;121(10):1875-1885

60 Welsh JD, Stalker TJ, Voronov R, et al. A systems approach to hemostasis: 1 . The interdependence of thrombus architecture and agonist movements in the gaps between platelets. Blood 2014;124(11):1808-1815

61 Du J, Fogelson AL. A two-phase mixture model of platelet aggregation. Math Med Biol 2018;35(02):225-256

62 Saltelli A, Ratto M, Andres T, Campolongo F, Cariboni J. Global Sensitivity Analysis: The Primer. West Sussex, United Kingdom: Wiley; 2008

63 Kahn ML, Nakanishi-Matsui M, Shapiro MJ, Ishihara H, Coughlin SR. Protease-activated receptors 1 and 4 mediate activation of human platelets by thrombin. J Clin Invest 1999;103(06): 879-887

64 Link KG, Stobb MT, Sorrells MG, et al. A mathematical model of coagulation under flow identifies factor $\mathrm{V}$ as a modifier of thrombin generation in hemophilia A. J Thromb Haemost 2020; 18(02):306-317

65 Shen L, Dahlbäck B. Factor V and protein S as synergistic cofactors to activated protein $\mathrm{C}$ in degradation of factor VIIIa. J Biol Chem 1994;269(29):18735-18738

66 Duckers C, Simioni P, Spiezia L, et al. Low plasma levels of tissue factor pathway inhibitor in patients with congenital factor $\mathrm{V}$ deficiency. Blood 2008;112(09):3615-3623

67 Peraramelli S, Thomassen S, Heinzmann A, et al. Role of exosite binding modulators in the inhibition of Fxa by TFPI. Thromb Haemost 2016;115(03):580-590 